\title{
Annihilating polynomials of excellent quadratic forms
}

\author{
KLAAS-Tido RüHL
}

\begin{abstract}
If $\varphi$ is an excellent form, then it is possible to use the dimensions of the higher complements of $\varphi$ to obtain an annihilating polynomial of $\varphi$ of low degree. The main result of this paper is the construction of such a polynomial with the help of methods from the theory of generic splitting of quadratic forms.
\end{abstract}

Mathematics Subject Classification (2000). Primary 11E81; Secondary 16R99.

Keywords. Quadratic form, annihilating polynomial.

1. Introduction. In [5] Lewis showed that for each $n \in \mathbb{N}_{0}$ the polynomial

$$
\mathbb{Z}[X] \ni P_{n}:= \begin{cases}X\left(X^{2}-2\right) \cdots\left(X^{2}-(n-2)^{2}\right)\left(X^{2}-n^{2}\right) & \text { for } n \text { even } \\ \left(X^{2}-1\right)\left(X^{2}-3^{2}\right) \cdots\left(X^{2}-(n-2)^{2}\right)\left(X^{2}-n^{2}\right) & \text { for } n \text { odd }\end{cases}
$$

annihilates every $n$-dimensional quadratic form over an arbitrary field $K$. Since then a number of classes of quadratic forms have been identified for which annihilating polynomials of significantly lower degree can be constructed, e.g. positive forms and trace forms. An extensive survey of the currently known results about annihilating polynomials of quadratic forms can be found in [6].

In this paper we will consider the class of excellent forms. Let $\varphi$ be an excellent form of dimension $n$ over $K$, let $h$ be its height, and for $j=0, \ldots, h$ let $n_{j}$ be the dimension of the $j$-th complement of $\varphi$. We will show that

$$
\mathbb{Z}[X] \ni E_{n}:= \begin{cases}X\left(X^{2}-n_{h-1}^{2}\right) \cdots\left(X^{2}-n_{1}^{2}\right)\left(X^{2}-n^{2}\right) & \text { for } n \text { even } \\ \left(X^{2}-1\right)\left(X^{2}-n_{h-1}^{2}\right) \cdots\left(X^{2}-n_{1}^{2}\right)\left(X^{2}-n^{2}\right) & \text { for } n \text { odd }\end{cases}
$$

The author gratefully acknowledges support from the Swiss National Science Foundation, grant no. 200020-109174/1 (project leader: E. Bayer-Fluckiger). 
annihilates $\varphi$. If $\varphi$ is isotropic, then we can prove this just by using the fact that $\varphi$ is a Pfister neighbour of a hyperbolic Pfister form. If $\varphi$ is anisotropic we will use methods from the theory of generic splitting of quadratic forms.

2. Notation and Basic Facts. The reader is expected to be familiar with the algebraic theory of quadratic forms and the theory of generic splitting of quadratic forms. An introduction to quadratic forms can be found in [7], [8] or [4]. The theory of generic splitting of quadratic forms was introduced in [2].

We define the set of natural numbers $\mathbb{N}$ not to contain 0 . If we need 0 as a natural number we use the notation $\mathbb{N}_{0}$. In all of this paper $K$ will be a field with $\operatorname{char}(K) \neq 2$, and we only consider non-degenerate quadratic forms over $K$. Often we will simply use the notion "form" when we mean a quadratic form.

Let $\varphi$ be a quadratic form over $K$. We denote by $\operatorname{dim}(\varphi)$ the dimension of the quadratic space over $K$ corresponding to $\varphi$. If $\psi$ is another quadratic form over $K$, then we write $\varphi \perp \psi$ and $\varphi \otimes \psi$ for the orthogonal sum and the tensor product of $\varphi$ and $\psi$. In case there exists a form $\chi$ over $K$ such that $\varphi \cong \psi \perp \chi$ we call $\psi$ a subform of $\varphi$. For $n \in \mathbb{N}_{0}$ the $n$-fold sum of $\varphi$ will be denoted by $n \times \varphi$. If $\varphi=\left\langle a_{1}, \ldots, a_{n}\right\rangle$ with $a_{1}, \ldots, a_{n} \in K^{*}$, then $a \varphi:=\left\langle a a_{1}, \ldots, a a_{n}\right\rangle$ for $a \in K^{*}$. In particular, if $a=-1$, we write $-\varphi:=\left\langle-a_{1}, \ldots,-a_{n}\right\rangle$. For $a_{1}, \ldots, a_{k} \in K^{*}$ we use the notation $\left\langle\left\langle a_{1}, \ldots, a_{k}\right\rangle\right\rangle$ for the $k$-fold Pfister form $\bigotimes_{i=1}^{k}\left\langle 1, a_{i}\right\rangle$. If there exists some $a \in K^{*}$ such that $\varphi \cong a \psi$ we say that $\varphi$ and $\psi$ are similar. We denote by $\widehat{W}(K)$, respectively $W(K)$, the Witt-Grothendieck ring, respectively Witt ring, of $K$. We write $[\varphi]$ for the isometry class of $\varphi$ in $\widehat{W}(K)$, and if $\varphi$ is isometric to $\psi$ we use the notation $\varphi \cong \psi$. In the case that $\varphi \cong n \times\langle 1\rangle$ we simply write $n$ instead of $[n \times\langle 1\rangle]$ for the isometry class of $\varphi$ in $\widehat{W}(K)$. The sum respectively product of $[\varphi]$ and $[\psi]$ in $\widehat{W}(K)$ will be denoted by $[\varphi]+[\psi]$ respectively $[\varphi][\psi]$ (or $[\varphi] \cdot[\psi])$. For an arbitrary element $[\varphi]-[\psi] \in \widehat{W}(K)$ we can extend the notion of dimension by setting $\operatorname{dim}([\varphi]-[\psi]):=\operatorname{dim}(\varphi)-\operatorname{dim}(\psi)$. If the forms $\varphi$ and $\psi$ represent the same element in $W(K)$ they are called equivalent, and we write $\varphi \sim \psi$. For an element $a \in K^{*}$ its class in the square class group $K^{*} /\left(K^{*}\right)^{2}$ will be denoted by $\bar{a}:=a\left(K^{*}\right)^{2}$.

If $L$ is a field extension over $K$, then we write $\varphi_{L}$ for the form $\varphi$ considered as a quadratic form over $L$. Most important in this paper is the case that $L$ is the function field $K(\varphi)$ of $\varphi$. To be exact, if $\operatorname{dim}(\varphi) \geq 2$ and $\varphi \neq\langle 1,-1\rangle$ we denote by $K(\varphi)$ the function field of the quadric defined by $\varphi$, otherwise we set $K(\varphi):=K$. Now consider a generic splitting tower $K_{0}:=K \subset K_{1} \subset \cdots \subset K_{h}$ of $\varphi$ (see [3, Section 5]), then $h$ is called the height of $\varphi$. For $j=0, \ldots, h$ we set $\varphi_{j}:=\left(\varphi_{K_{j}}\right)_{\text {an }}$, where $\left(\varphi_{K_{j}}\right)_{\text {an }}$ denotes the anisotropic kernel of $\varphi_{K_{j}}$, and call $\varphi_{j}$ the $j$-th anisotropic kernel of $\varphi$. The form $\varphi$ is a Pfister neighbour if there exists a Pfister form $\tau$ over $K$ such that $\varphi$ is similar to a subform of $\tau$ and $\operatorname{dim}(\varphi)>\frac{1}{2} \operatorname{dim}(\tau)$. If $\chi$ is a form over $K$ and $a \in K^{*}$ such that $a \tau \cong \varphi \perp \chi$, then $\chi$ is called the complement of $\varphi$. We say that $\varphi$ is excellent if there exists a sequence 
$\psi_{0}:=\varphi, \psi_{1}, \ldots, \psi_{h}$ of forms over $K$ such that $\operatorname{dim}\left(\psi_{h}\right) \leq 1$ and $\psi_{i-1}$ is a Pfister neighbour with complement $\psi_{i}$ for $i=1, \ldots, h$. The $\psi_{i}$ are the higher complements of $\varphi$. In this setting, if $\varphi$ is anisotropic, it is well-known that $\left(\psi_{j}\right)_{K_{j}} \cong(-1)^{j} \varphi_{j}$ for $j=0, \ldots, h$ (see [3, Proposition 7.9]).

Definition 2.1. Let $R$ be a unitary, commutative ring, and let $\iota: \mathbb{Z} \rightarrow S$ be the canonical homomorphism defined by $\iota(1)=1_{S}$. If $P=a_{n} X^{n}+\ldots a_{1} X+a_{0} \in \mathbb{Z}[X]$ is a polynomial and $x \in S$, then $P$ is called an annihilating polynomial of $x$ if

$$
P(x):=\iota\left(a_{n}\right) x^{n}+\cdots+\iota\left(a_{1}\right) x+\iota\left(a_{0}\right)=0 .
$$

Usually we will omit the $\iota$ and just write $a s:=\iota(a) s$ for $a \in \mathbb{Z}$ and $s \in S$.

Let $G:=K^{*} /\left(K^{*}\right)^{2}$ be the square class group of $K$. We use Hurrelbrink's approach in [1] to construct annihilating polynomials for elements of the group $\operatorname{ring} \mathbb{Z}[G]$ :

Consider the dual group

$$
\widehat{G}:=\left\{\chi: G \rightarrow S^{1} \mid \chi \text { is a group homomorphism }\right\}
$$

of $G$, where $S^{1} \subset \mathbb{C}$ is the unit circle. Then each character $\chi \in \widehat{G}$ can be extended to a $\mathbb{Z}$-algebra homomorphism

$$
\underline{\chi}: \quad \mathbb{Z}[G] \longrightarrow \mathbb{C}, \quad \sum_{g \in G} z_{g} g \longmapsto \sum_{g \in G} z_{g} \chi(g) .
$$

For $f \in \mathbb{Z}[G]$ the set $S_{f}:=\{\underline{\chi}(f) \mid \chi \in \widehat{G}\}$ is finite by [1, Observation 1.2], and it is easy to see that it is a subset of $\mathbb{Z}$. This allows us to define the polynomial

$$
P_{f}:=\prod_{\underline{\chi}(f) \in S_{f}}(X-\underline{\chi}(f)) \in \mathbb{Z}[X],
$$

which by $[1$, Theorem 1.3] is an annihilating polynomial of $f$.

Now consider the canonical surjection $\pi_{1}: \mathbb{Z}[G] \rightarrow \widehat{W}(K)$ defined by

$$
\overline{a_{1}}+\cdots+\overline{a_{n}} \longmapsto\left\langle a_{1}, \ldots, a_{n}\right\rangle .
$$

Definition 2.2. An element $f=\sum_{g \in G} z_{g} g \in \mathbb{Z}[G]$ is called a preform if $z_{g} \geq 0$ for all $g \in G$. The integer $n:=\sum_{g \in G} z_{g}$ is the dimension of $f$, and we write $\operatorname{dim}(f):=n$. If $f=\overline{a_{1}}+\cdots+\overline{a_{n}}$, and if $\varphi$ is a quadratic form over $K$, then $f$ is called a preform of $\varphi$ if we have $\varphi \cong\left\langle a_{1}, \ldots, a_{n}\right\rangle$ or equivalently $\pi_{1}(f)=[\varphi]$.

Definition 2.3. Let $\varphi$ be a quadratic form over $K$. A polynomial $P \in \mathbb{Z}[X]$ is called an annihilating polynomial of $\varphi$ if it is an annihilating polynomial of $[\varphi] \in \widehat{W}(K)$.

Corollary 2.4. If $f \in \mathbb{Z}[G]$ is a preform of a quadratic form $\varphi$ over $K$, then $P_{f}$ is an annihilating polynomial of $\varphi$. 
Proof. We have

$$
P_{f}([\varphi])=P_{f}\left(\pi_{1}(f)\right)=\pi_{1}\left(P_{f}(f)\right)=\pi_{1}(0)=0 .
$$

Remark 2.5. If $f \in \mathbb{Z}[G]$ is a preform of an arbitrary form $\varphi$ over $K, \operatorname{dim}(\varphi)=n$, then it is easy to see that $S_{f} \subset\{-n,-n+2, \ldots, n-2, n\}$ (see [1, Observation $2.2]$ ). We thus obtain the result first presented by Lewis in [5], that the polynomial

$$
P_{n}:=(X+n)(X+n-2) \cdots(X-n+2)(X-n) \in \mathbb{Z}[X]
$$

annihilates all $n$-dimensional quadratic forms over $K$.

\section{Main Result.}

Lemma 3.1. If $\varphi$ and $\psi$ are quadratic forms over $K$ with $\varphi \sim \psi$, and if $P \in \mathbb{Z}[X]$ is an annihilating polynomial of $\varphi$, then $P([\psi]) \sim 0$.

Proof. Let $\pi_{2}: \widehat{W}(K) \rightarrow W(K)$ be the natural projection. Then

$$
0=\pi_{2}(P([\varphi]))=P\left(\pi_{2}([\varphi])\right)=P\left(\pi_{2}([\psi])\right)=\pi_{2}(P([\psi]))
$$

or equivalently $P([\psi]) \sim 0$.

Proposition 3.2. Let $\varphi$ be a Pfister neighbour of dimension $n>0$ with complement $-\psi$ over $K$, and let $a \in K^{*}$ and $\tau$ be a Pfister form such that $\varphi \perp-\psi \cong a \tau$. If $Q \in \mathbb{Z}[X]$ is an annihilating polynomial of $\psi$, then $P:=Q \cdot\left(X^{2}-n^{2}\right)$ is an annihilating polynomial of $\varphi$. In the case that $a \in\left(K^{*}\right)^{2}$ (in particular if $\varphi$ is isotropic) the polynomial $\widetilde{P}:=Q \cdot(X-n)$ suffices.

Proof. If $\varphi$ is isotropic, then $\tau$ is isotropic as well and therefore hyperbolic. Hence $\varphi \sim \psi$. By the previous lemma $Q([\varphi]) \sim 0$, and therefore

$$
Q([\varphi]) \cdot[\varphi] \sim 0 \sim Q([\varphi]) \cdot n .
$$

The claim follows since $\operatorname{dim}(Q([\varphi]) \cdot[\varphi])=\operatorname{dim}(Q([\varphi]) \cdot n)$.

Now let $\varphi$ be anisotropic. Since $\varphi_{K(\varphi)} \sim \psi_{K(\varphi)}$, we obtain $Q\left(\left[\varphi_{K(\varphi)}\right]\right) \sim 0$ from the previous lemma. A result by Knebusch ([2, Example 4.1]) states that $Q\left(\left[\varphi_{K(\tau)}\right]\right) \sim 0$. Hence by $[2$, Lemma 4.4$]$ there exists an element $x \in \widehat{W}(K)$ such that $Q([\varphi])=x \cdot[\tau]$. Now $a \varphi \subset \tau$. Since $b \tau \cong \tau$ for all $b \in K^{*}$ represented by $\tau$, it follows that $a \varphi \otimes \tau \cong n \times \tau$. If $a \in\left(K^{*}\right)^{2}$, then

$$
Q([\varphi]) \cdot([\varphi]-n)=x \cdot[\tau] \cdot([\varphi]-n)=x \cdot([\varphi \otimes \tau]-[n \times \tau])=x \cdot 0=0 .
$$

Otherwise, if $a$ is not a square, we still obtain

$$
\begin{aligned}
Q([\varphi]) \cdot\left([\varphi]^{2}-n^{2}\right) & =x \cdot[\tau] \cdot\left([\varphi]^{2}-n^{2}\right)=x \cdot\left([\tau] \cdot[a \varphi]^{2}-[\tau] \cdot n^{2}\right) \\
& =x \cdot\left([a \varphi \otimes a \varphi \otimes \tau]-\left[n^{2} \times \tau\right]\right)=x \cdot 0=0 .
\end{aligned}
$$


Theorem 3.3. Let $\varphi$ be an excellent form of height $h \in \mathbb{N}_{0}$ over $K$, let $\varphi=$ $\psi_{0}, \psi_{1}, \ldots, \psi_{h}$ be the sequence of its higher complements, and for $j=0, \ldots, h$ set $n_{j}:=\operatorname{dim}\left(\psi_{j}\right)$. Then

$$
\mathbb{Z}[X] \ni E_{n}:= \begin{cases}X\left(X^{2}-n_{h-1}^{2}\right) \cdots\left(X^{2}-n_{1}^{2}\right)\left(X^{2}-n^{2}\right) & \text { for } n \text { even } \\ \left(X^{2}-1\right)\left(X^{2}-n_{h-1}^{2}\right) \cdots\left(X^{2}-n_{1}^{2}\right)\left(X^{2}-n^{2}\right) & \text { for } n \text { odd }\end{cases}
$$

annihilates $\varphi$.

Proof. We proceed by induction on $h$. If $h=0$ and $\operatorname{dim}(\varphi)=0$, then indeed $E_{0}=X$ annihilates $\varphi=0$. In the case $\operatorname{dim}(\varphi)=1$ we have $\varphi=\langle a\rangle$ for some $a \in K^{*}$, and $[a]^{2}=1$. This shows, that $E_{1}=X^{2}-1$ annihilates $\varphi$.

Now let $h>0$. By induction $E_{n_{1}}$ annihilates the excellent form $\psi_{1}$. Since $\psi_{1}$ is the complement of $\varphi$ it follows from the previous lemma that $E_{n_{1}} \cdot\left(X^{2}-n^{2}\right)=E_{n}$ annihilates $\varphi$.

Remark 3.4. It is well-known that the dimensions of the higher complements and the height of an excellent form $\varphi$ only depend on the dimension of $\varphi$ and can be explicitly calculated with the help of certain recursive functions (see [3, Corollary 7.11]).

Remark 3.5. Let $\varphi$ be similar to a $k$-fold Pfister form, $k \in \mathbb{N}_{0}$. A consequence of proposition 3.2 is the well-known result that $\varphi$ can be annihilated by $X\left(X^{2}-2^{2 k}\right)$ $=X\left(X-2^{k}\right)\left(X+2^{k}\right) \in \mathbb{Z}[X]$. If $\varphi$ is a $k$-fold Pfister form it suffices to take the polynomial $X\left(X-2^{k}\right)$.

There exists an easy proof of this result that only uses corollary 2.4: Let $\varphi$ be similar to a $k$-fold Pfister form. Then there exist $g_{0}, g_{1}, \ldots, g_{k} \in G:=K^{*} /\left(K^{*}\right)^{2}$ such that $f=g_{0} \cdot\left(1+g_{1}\right) \cdots\left(1+g_{k}\right) \in \mathbb{Z}[G]$ is a preform of $\varphi$. Since for every $\chi \in \widehat{G}$ the map $\chi$ is a homomorphism of rings, and since $\chi\left(1+g_{i}\right) \in\{0,2\}$ for all $i=1, \ldots, k$, it follows that $\underline{\chi}(f) \in\left\{-2^{k}, 0,2^{k}\right\}$ and therefore $S_{f} \subset\left\{-2^{k}, 0,2^{k}\right\}$. If $g_{0}=1$ we even get $S_{f} \subset\left\{0,2^{k}\right\}$.

\section{References}

[1] J. Hurrelbrink, Annihilating polynomials for group rings and Witt rings. Canad. Math. Bull. 32(4), 412-416 (1989).

[2] M. Knebusch, Generic splitting of quadratic forms I. Proc. London Math. Soc. 33, 65-93 (1976).

[3] M. Knebusch, Generic splitting of quadratic forms II. Proc. London Math. Soc. 34, 1-31 (1977).

[4] T.-Y. LAM, Introduction to Quadratic Forms over Fields. American Mathematical Society, 2005.

[5] D. W. LEWIS, Witt rings as integral rings. Invent. Math. 90, 631-633 (1987). 
[6] D. W. Lewis, Annihilating polynomials for quadratic forms. Int. J. Math. Math. Sci. 27(7), 449-455 (2001).

[7] A. Pfister, Quadratic Forms with Applications to Algebraic Geometry and Topology, London Math. Soc. Lecture Note Series 217. Cambridge University Press, 1995.

[8] W. Scharlau, Quadratic and Hermitian Forms, Grundlehren der math. Wissenschaften 270. Springer-Verlag Berlin Heidelberg New York Tokyo, 1985.

KlaAs-Tido RüHL, EPFL SB IMB CSAG, MA C3 575 (Batiment MA), Station 8, CH-1015 Lausanne, Switzerland

e-mail: klaas-tido.ruhl@epfl.ch

Received: 23 April 2007 